ox or some such animal, and I shall never forget the courteous way in which an eminent palæontologist assured him that the specimen should really go to the Museum at Bloomsbury, since a fossil would show only the bones, and not solidified flesh. The public certainly gets all it pays for, and more ; but it could be much better served if it would pay enough to bring out the latent possibilities of the museum, which are doubtless greater than any of us can yet clearly imagine.

University of Colorado.

\section{Spiranthes Autumnalis.}

IN the summer holiday of $\mathrm{x} 92 \mathrm{r}, \mathrm{Mr}$. Mayland and I were astonished to find in the woods round Carrbridge, Inverness-shire, very sporadically but at two stations about a mile apart, specimens of the small orchid, Spiranthes autumnalis. We took some, and for two or three days their characteristic scent and spiral spikes interested our table in the hotel. I regret now that we did not preserve specimens : but I am pretty sure we were not mistaking the identity of the plant, as it was repeatedly the subject of remark, and I have known it since I was a boy.

I mention this non-recorded record because Sir Herbert Maxwell, who in September I920 wrote to NATURE (vol. I06, p. 79), telling of a similar experience on Lower Spey-side ; but in a later letter (vol. Io6 p. 409), he expressed some uncertainty as to the identity of his plant as apart from Goodyera repens. Now when so acute an observer as Sir Herbert has arrived independently at the same conclusion as we did, I think the probability is strong that both diagnoses were correct, and that, though the specimens were not preserved as evidence, $S$. autumnalis has been found in an unexpected, non-calciferous locality.

Mr. Mayland tells me he sought it again in the following summer without result. F. O. Bower.

The University, Glasgow, January I 7 .

\section{The Scattering of X-Rays in Liquids.}

IN various notes published last year I dealt with the scattering of light in transparent media, and showed that its study initiated by the late Lord Rayleigh in his theory of the colour of the sky has other fascinating applications in the explanation of the colour of the sea and other transparent waters, and of the colour of ice on glaciers. The thermodynamic theory of "fluctuations" developed by Smoluchowski and Einstein formed the starting-point in the discussions, but I was careful to emphasise the important complications arising from the anisotrophy of the molecules in fluid media and showed how the necessary corrections in Einstein's theory may be made. A considerable measure of success was attained in attempting to correlate the behaviour of substances in the liquid and gaseous states in this respect, and in predicting the effects due to alterations of temperature and pressure. The study of the changes in the intensity and states of polarisation of the scattered light in passing from the liquid to the solid crystalline state and their explanation forms another important line of inquiry in which some progress has also been made.

The purpose of the present note is to point out the relation between the optical effects referred to above and the very interesting recent work of Keesom and Smedt, who have obtained Laue photographs of various liquids traversed by a homogeneous pencil of X-rays (Proc. Roy. Soc. Amsterdam, I922, page I I9), and the similar work by Hewlett (Physical Review, December I922, page 702), who used the ionisation method. Keesom and Smedt found that many of the liquids studied gave a well-marked diffraction ring at a considerable angle with the direct pencil. With liquid oxygen and argon, the first ring was formed at an angle of $27^{\circ}$. A weak second ring was also observed at $46^{\circ}$ with oxygen, and at $49^{\circ}$ with argon. With water, on the other hand, the second ring was very broad and diffuse and practically abutted on the first.

Keesom and Smedt have attempted to explain their results by various special assumptions regarding the relative positions of the neighbouring molecules, while Hewlett suggests that liquids possess something of a crystal structure. To the present writer it appears that the experimental results may be explained without any such special assumptions. As in the optical case, the liquid molecules may be regarded as the diffracting centres which are arbitrarily orientated and distributed uniformly in space subject only to such variations as give rise to density fluctuations in accordance with the Einstein-Smoluchowski formula,

$$
\overline{(\Delta \rho)^{2}}=\frac{\mathrm{RT} \beta}{\mathrm{V}} \cdot \rho_{0}^{2}
$$

where $\rho_{0}$ is the mean density $\overline{(\Delta \rho)^{2}}$ the mean square of its fluctuations, $\mathrm{R}$ the gas constant, $\mathrm{T}$ the absolute temperature, $\beta$ the compressibility of the liquid, and $\mathrm{V}$ the elementary volume under consideration. When traversed by a honogeneous pencil of X-rays the wave length of which is smaller than the average molecular distance, such a structure must give rise to diffraction rings which are more or less well defined according as the fluctuations of density are small or large. If in the expression for the density fluctuation, we take $\mathrm{V}$ to be a small cube with a molecule at each of its corners, the average fluctuation in its size and the resulting weakening of the diffraction pattern can be calculated somewhat on the same lines as in Debye's theory of thermal effect in X-ray reflection by crystals. In Keesom and Smedt's experiments, the low temperature in the case of liquid oxygen and argon, and the consequently diminished fiuctuations of density must have helped in improving the definition of the diffraction ring of the second order.

$$
\text { C. V. Raman. }
$$

\section{"Artificial" Vertical Beam.}

THE vertical beam through a low sun is generally referred to the reflection of sunlight from the basal surfaces of thin plates of ice which are falling through the atmosphere with their crystal axes vertical and horizontal. It has been the writer's good fortune to examine such reflections from individual "plates" that were slowly falling within a metre or so of the observer. Most of the plates were asymmetric portions of flat crystal growths, and they. spun rapidly as they fell, with a motion resembling that of a falling maplekey. In this case, the vertical beam was observed to spread out slightly as it receded from the sun, and the angle subtended by the edges of the beam was obviously the complement of the vertical angle of the " cone" swept out by the rapidly rotating, but slowly falling flake.

An interesting " artificial " example of (probably) this phenomenon, was noted by many observers at the burning of two buildings at the Sydenham Military Hospital at Kingston, Ontario, on the night of January 3, I923. The structures burned fiercely in the brisk north-east wind and lit up the snow-covered country for miles around. The unusual brightness may be judged from the credible report that people more than a mile from the fire easily read newsprint by its light. A very light snow-fall was barely noticeable from time to time during the evening. Out of the glow of the fire-lit smoke clouds there appeared to rise a vertical parallel beam of light, that with varying distinctness was visible for the three or four hours 